\title{
A Survey of Light Emitting Diode (LED) Display Board
}

\author{
Gowrishankar Kasilingam*, Mritha Ramalingam and Chandra Sekar \\ Faculty of Engineering \& Computer Technology, AIMST University, Kedah, Malaysia; \\ gowri200@yahoo.com, mritha88@gmail.com
}

\begin{abstract}
In this paper an overview of different Light Emitting Diode (LED) display boards is presented. The enhanced LED plugs in all the loop holes in the existing product. Global Service for Message (GSM) based LED Display Board is a model for displaying notices or messages at places that require real-time noticing, by sending messages in the form of Short Message Service (SMS) through mobile. This paper proposes an idea to develop a sign board in which the messages are continuously running, where it empowers the user to change the messages using SMS instantaneously and also desk bound device such as PC or laptop. It has a dual system in terms of changing message display. It uses dual power supply such as solar power and Alternative Current (AC) power which creates the ability to work at any situation. The new design includes a motion detector which switches off the system automatically after working hours and will switch on automatically if any motion is sensed by the motion detector after the programmed working hours. This paper is a comprehensive overview of the literature and it does not contain implementation results.
\end{abstract}

Keywords: Dual Power Supply, LED Display, Motion Detection, Scrolling Message, Wireless

\section{Introduction}

An information display is a way of providing information and/or is used as an object for promotion. It can be seen in a form of cardboard or tarpaulin at stores/shops, streamers and electronic display devices. But the advent of new technologies made the information in the form of an electronic display in the world of advertisements and promotions. The ability to display a short message can be useful application to be available for any business. A LED display board is perfect for this application. It can be used for both indoor and outdoor which makes it universal fit for any business or event. The LED display board is very efficient and cost effective way to spread messages to thousands of people, without any personal contact or door-to-door sales. LED is a solid state light source with several attractive properties for display application. It is chosen as the main component for displaying messages because, today LED is the most energy efficiency example as compared to incandescent light bulb. A LED light emit very little heat and saves a lot of energy, as 98 percent of the energy used by a traditional incandescent light can be lost as heat energy instead of light energy. A GSM based LED display board is a device for displaying messages by sending the message in the form of SMS through mobile.

Globally, over billion people have recognized the need of GSM for mobile phone applications. The high frequency, $900 \mathrm{MHz}$ and international roaming of the GSM makes the mobile phone users to recognize the technology world wide. Because of the digitalised signals and speech clarity, the GSM technology is considered as third generation (3G) mobile communication system.

A motion detector is a device that detects moving objects, particularly people. A motion detector is often integrated as a component of a system that automatically performs a task or alerts a user of motion in an area. Motion detectors form a vital component of security, automated lighting control, home control, energy efficiency,

${ }^{*}$ Author for correspondence 
and other useful systems. This paper proposes a LED display board with dual power supply such as solar and AC power and changing the displaying message either by laptop or SMS through mobile phones. This paper is also intended to incorporate a motion detector which acts as a switch in the display board.

\section{Literature Review}

In the mid 1990s, cost-effective traffic light lamps using LEDs were developed. Prior to this, traffic lights were designed using incandescent or halogen light bulbs. Unlike the incandescent-based lamps, which use a single large bulb, the LED based lamps consist of an array of LED elements, arranged in various patterns. When viewed from a distance, the array appears as a continuous light source.

A wireless system was designed wherein the display board need not be reprogrammed to display a new message. This paper was aimed to develop a mobile sign board which makes the user to change the scrolling message using SMS service instantaneously. The user can update it even from a remote distant. Once read, the SMS is deleted making entry for next incoming SMS ${ }^{1}$.

One of the main interesting applications that have led the use of embedded system in communication is the public addressing system. The audio or video systems like public announcement system, CCTV and programmable sign boards are generally hardwired and hard to enlarge. These limitations can be trounced by the use of wireless interface such as GSM. Currently LED message scrolling displays are attracting many researchers in the community ${ }^{2}$. A LED scrolling message display system using Android application was designed. This system used Bluetooth technology to communicate from Android phone to LED display board ${ }^{3}$.

Message displaying system using LED has become a significant part of many applications concentrating on brightness and power saving concept. The low power consumption and longer life span of LED ensures that the number of applications using them will increase further in near future. This message displaying system uses $5 \mathrm{~mm}$ LED and microcontroller M68HC11. This system can display 8 alphanumeric characters in one time whereby the arrangement of LEDs is using matrix display with less connection needed. Multiplexing and matrix addressing method is applied to control the turn on and off of LED in that particular time ${ }^{4}$.

Because of less maintenance, the wireless communication is identified as a perfect solution for home network, and its great demand can be recognized in data transfer using mobile applications. A simple GSM technology to receive and display the messages was used in $^{5}$. Looking into the present trend of information transfer, it is seen that vital notice take time to be displayed on the displaying boards. The advancement in the technologies related to wireless communication has led to the emergence of several engineering designs to aid the human requirements. Kamboj and $\mathrm{Abrol}^{6}$ presents a combination of wireless technology with LED display boards to overcome the difficulties faced by the existing message display modules using any wired entries like computer, keyboard or less distance remote control. The message is sent through a cell-phone which is accepted by the GSM module SIM 300 (master). Number authentication is done by AT89S52 microcontroller and the stored numbers in EEPROM is compared with the incoming number. The message will be valid only after the incoming cell phone number is validated. Authentication result is displayed on LCD. Further the same SMS is itself sent by GSM module (master) to multiple LED display boards which are connected via different GSM modules (slaves).

$\operatorname{In}^{7}$ a system to display the message received by the cell or a modem was proposed. Microcontroller controls the system using authentication and attention (AT) commands. This system is easy, robust to use in normal life by any-one at any place.

A LED display control system can also be designed using advanced reduced instruction set computer machines and field programmable gate array ${ }^{8}$. According to module structure characteristics of the Red, Green, Blue (RGB) three colours LED display and the dynamic scanning display of the LED display. This system supports texts and pictures to display with full colour LED screen and a remote data transmission.

A microcontroller based display system with the size of six $32 \times 32$ dot matrix Chinese characters by a dynamic scan mode is discussed in ${ }^{9}$. The system can be divided into two small display screens which can display 24 Chinese characters. The display contents and scrolling speed can be adjusted according to user requirements.

LED display notice board is an economical solution for displaying messages with various changing effects and can be applicable in shops, restaurants, pharmacies, etc. These boards can be large LED panels or LED dot matrix displays which could be in mono, bi, and multicolour. The power consumption of these boards is less. This can display static and dynamic messages ${ }^{10}$. 
A radio frequency system was designed, that consists of spinning LED to display the messages send by the user at the handheld device. If there are no inputs from the user for the system, the message that is programmed in the microcontroller will be displayed continuously. The vertical display uses 7 LEDs only. In order to view the displayed message the LED motor has to rotate faster ${ }^{11}$. A circular LED display works by scanning mechanically and displaying the characters digitally using only 7 LEDs $^{12}$.

\section{Problem Identification}

Based on the papers reviewed, we identified some drawbacks which induced us to concentrate or designing a new enhanced LED display board. A common drawback that exists is, none of the designs can display more than a message at a time at big scale. Another common drawback found in these systems is, the display board functions using only one type of power supply, which is either AC power or solar power. Besides, in terms of changing message the existing designs, can be able to accommodate only one way of changing message which is either by GSM or by laptop. None of the above system is able to function without manual switching on and off the system. It requires man power to handle the display board. The above inventions will not function if power shortage was to take place nor changes in weather occur since each system is only bound to one type of power supply. Therefore, there is no alternative means of measure for unforeseen circumstances. Next, the systems are designed with either type of message changing option, which means incase of transmission interferences, the GSM based technology will not be able to function, while for the laptop based, incase if the system breakdown, the message cannot be changed either. When two or more different messages are displayed, the time taken to view the first message again will be longer. This is very cumbersome for people to obtain information faster due to the prolonged time taken to repeat the messages once again. Besides the system requires man power to operate, which means carelessness of man can either lead to wastage of current if in case the system is not switched off when not needed or if forgotten to switch on, the message will not be displayed. This clearly explains that the past inventions are not pre-planned nor has far sighted visions.

\section{The Proposed Work}

The proposed system is designed in such a way that it works based on solar power as well as AC power. On a daily basis, the electricity is generated using solar panel and the power is stored at the power bank. However, occurrence of weather change where insufficient solar energy is generated, the system is able to function as normal, using AC power. Another advantage of the design is the ability to change the message of the display board either by GSM technology as well as desk bound method, so it has dual capability in terms of changing messages which is yet another not existing feature in display boards. This assures that even in the case of signal transmission interference during message transfer to GSM, the alternative pathway would be available through laptop means and vice versa. This system is quiet capable of displaying more than one message at a single time and provides information much faster than the existing technology and reduces the period of waiting to read again the first message. Because of this advantage, this system can be commercialised by renting out the second message display column to another user and at the same time, the key owner of the display board can display his/her message as well. The enhanced LED display board will also be designed with a motion detector. The function of motion detector is to act as a switch to automatically turn off the system when it is not needed. This is yet a unique feature added to the existing technology which reduces power wastage, unlike the existing technology where in case of negligence in switching off the system after the required period, the system continues to run until it is switched off manually. All of the above proposed features certainly uphold a far sighted vision which is why the new design is said to be enhanced than the current technology. Figure 1 shows the block diagram of the proposed LED display board.

\section{Conclusion}

This paper identifies the drawbacks that have been found in every existing work. As a result, by introducing the concept of enhanced LED display board, this paper explains by integrating features of dual power supply, dual option of changing message and inbuilt motion detector in the field of communication. Such collaborative powers and method of changing message had not been developed in a single device, and this paper marks the beginning of the new technology of combining two separate technologies under one roof. Therefore, this new enhanced display system makes our communication more efficient and faster. Certainly this model may be able to work under any circumstances with greater efficiency, as it does not need any man power to switch off the system 


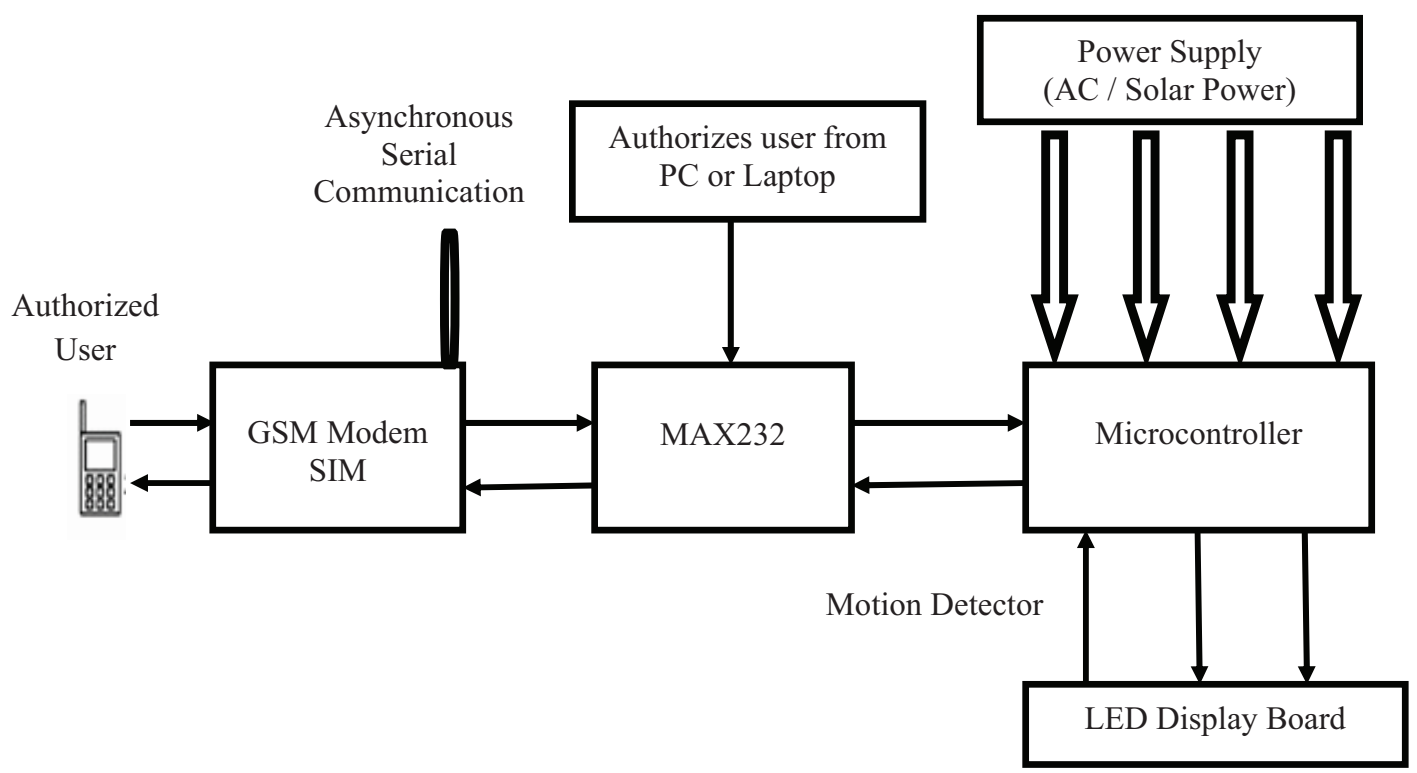

Figure 1. Block diagram of proposed system.

because it is an automated system. Besides, in this paper the user can display additional messages at a time. From this the user of this display board can use the board for rental out, notice board and promotion board. In a nutshell, this paper describes the new enhancement in LED display board which is highly efficient than the existing technology.

\section{Acknowledgement}

The authors acknowledge the AIMST University for the support and providing the research grant for finishing this work successfully.

\section{References}

1. Gupta H, Shukla P, Nagwekar A. GSM based LED scrolling Display Board. International journal of Students Research in Technology and Management. 2013; 1(3):278-91.

2. Ketkar PU, Tayade KP, Kulkarni AP,Tugnayat RM. GSM Mobile Phone Based LED Scrolling Message Display System. International Journal of Scientific Engineering and Technology. 2013; 2(3):149-55.

3. Sooxma Technology. Android Controlled Scrolling LED Message Display. Hyderabad, India.
4. Bin Zohedi FN. Wireless electronic notice board. Faculty of Electrical \& Electronics Engineering, University Malaysia Pahang; 2007.

5. Kumar P, Bharadwaj V, Pal K, Rathor NR, Mishra A. GSM based e-Notice Board: wireless communication. International Journal of Soft Computing and Engineering. 2012; 1-2(3):601-5.

6. Kamboj R, Abrol P. Design and development of GSM based multiple LED display boards. International Journal of Computer Applications. 2013; 71(18):40-6.

7. Dalwadi DC, Trivedi N, Kasundra A. Wireless notice board our real-time solution. National Conference on Recent Trends in Engineering \& Technology; 2011.

8. Ni X, Yan Z, Dan L, Zhou Y. The realization of led display system based on the embedded, department of computer engineering. Telkomnika. 2013; 11(5):2626-33.

9. Song Y, Feng Y, Ma J, Zhang X. Design of LED display control system based on AT89C52 single chip microcomputer. China, Journal of Computers. 2011; 6(4):718-24.

10. Wimalasena H. LED Notice Board; 2013.

11. Sarode A, Salunke DM, Shukla AL, Sonawadekar MM. Spinning LED display using radio frequency. 2012; International Journal of Computer Science and Informatics 2(4): 83-86.

12. Manihar SR, Dewangan KP, Dansena AK. The Design and construction of a low cost propeller LED display. Global Journal of Researches in Engineering-Electrical and Electronics Engineering. 2012; 12(4): 